\title{
Research on the Interaction Among Financial Markets in China, Hong Kong and the United States — Based on Modified CHMM Model
}

\author{
Li Tong, ${ }^{1, a}$, Zhou Sijia ${ }^{2, b}$, Qiu Xuelin ${ }^{3, c}$, Yang Xuanwei ${ }^{4, d}$ \\ ${ }^{1}$ Department of Business Administration, School of Management, Shenzhen University, Nanshan, Shenzhen, \\ China \\ ${ }^{2}$ Department of Business Administration, School of Management, Shenzhen University, Nanshan, Shenzhen, \\ China \\ ${ }^{3}$ Department of Business Administration, School of Management, Shenzhen University, Nanshan, Shenzhen, \\ China \\ ${ }^{4}$ Department of Business Administration, School of Management, Shenzhen University, Nanshan, Shenzhen, \\ China \\ a611994240@qq.com \\ b1914636546@qq.com \\ c2815342076@qq.com \\ d296748750@qq.com
}

\begin{abstract}
This paper takes the stock market, bond market and futures market price index returns rates in China, Hong Kong and the United States as research objects, and uses the modified CHMM model to study the interaction between different financial market price indexes. The empirical results show that the interaction between financial market price indexes is relatively close during the sample period; American stock market, American bond market and American futures market are the active initiators of interaction. American financial market has a stronger interactive influence on Hong Kong financial market than China, and China is the active initiator of interaction between China and Hong Kong.
\end{abstract}

Keywords: International financial market, interactive influence, modified CHMM model

\section{中国、香港和美国金融市场间交互影响研究——基于 动态影响模型}

李峡 ${ }^{1, a}$, 周思佳 ${ }^{2, b}$, 邱雪林 ${ }^{3, \mathrm{c}}$, 杨玄威 ${ }^{4, \mathrm{~d}}$

${ }^{1}$ 深圳大学管理学院工商管理系, 南山, 深圳, 中国

2 深圳大学管理学院工商管理系, 南山, 深圳, 中国

3 深圳大学管理学院工商管理系, 南山, 深圳, 中国

${ }^{4}$ 深圳大学管理学院工商管理系, 南山, 深圳, 中国

a611994240@qq.com

b1914636546@qq.com

c2815342076@qq.com

d296748750@qq.com

摘要

本文以中国、香港和美国的股市、债市和期货市场价格指数收益率为研究对象，采用动态影响模型对不同金 
融市场价格指数之间的交互影响进行研究。实证结果表明，样本期内金融市场价格指数之间的交互影响程度 较为密切; 美国股市、美国债市和美国期货市场是交互影响的主动发出者，美国金融市场对香港金融市场的 交互影响程度强于中国, 中国是中国和香港交互影响关系中的主动发出者。

关键词: 国际金融市场, 交互影响, 动态影响模型

\section{1. 引言}

随着经济一体化和金融全球化的发展，国际金 融市场间的资本与信息流动在不断增强，这使得国 际金融市场中各个金融市场之间的关系变得错综复 杂, 中国、香港和美国的经济联系近年来越来越紧 密, 任何一方的金融市场波动都会对其他方产生影 响, 而三者的金融市场关系不仅会影响投资者的资 产配置, 也会给各个国家和地区的宏观经济调控政 策的制定带来影响，那么系统地对三者金融市场间 的交互影响进行研究就显得十分重要。

纵观国内外现有研究, 对金融市场交互影响方 面的研究成果不少, 就研究对象而言大致分四类。

一是同一地区同一金融市场之间的交互影响 ${ }^{[1]}$ 。二 是同一地区不同金融市场之间的交互影响 ${ }^{[2]}$ 。三是 不同地区同一金融市场之间的交互影响 ${ }^{[3]}$ 。四是不 同地区不同金融市场之间的交互影响 ${ }^{[4]}$ 。

就研究方法而言, 现有的文献采用的研究方法 主要有四类。一是 VAR 模型及其扩展方法 ${ }^{[5]}$ 。二是 多元 GARCH 模型及其扩展方法 ${ }^{[6]}$ 。三是基于 Copula 函数模型的方法 ${ }^{[7]}$ 。四是基于变量间相关系数的相 关关系和溢出效应研究方法 ${ }^{[8]}$ 。在实际研究中, 通 常将以上四类方法结合使用，这类方法虽有所差异， 但都基于回归的方法, 回归检验虽有一定的效果, 但由于会受到序列自相关性、共线性等影响, 有可 能得到伪结论, 且只能揭示出线性关系。

通过以上分析可以发现, 现有研究虽取得了一 定的研究成果, 但仍存在一些局限性: 第一, 研究 对象大多为同一地区不同金融市场和不同地区同一 金融市场之间交互影响的研究, 现有的对不同地区
不同金融市场之间交互影响的研究非常少且基本是 两个地区多个市场, 很少研究两个以上地区的多个 市场之间的关系; 第二, 目前的研究方法灵活性较 差, 对数据要求较高, 要求数据稳定且满足线性关 系，但是存在不能满足金融时间序列非线性的问题， 除此之外数据的处理及计算过程也较为复杂。鉴于 此，本文采用动态影响模型对中国、香港、美国的 股票市场、债券市场、期货市场间的交互影响进行 研究，这不仅可以解决不能满足金融时间序列非线 性的问题，还能捕捉到不同波动情况下市场之间非 线性、非对称的动态关系，更好地对不同地区不同 金融市场间的交互影响关系进行分析。

\section{2. 模型介绍}

动态影响模型由 MIT 提出, 是一种由 HMM 模型 演变而来的机器学习算法, 是指实体的状态受其网 络邻居状态的影响并随之变化, 由相互作用与影响 的实体组成, 每个实体都有基于观测状态和隐藏状 态的时间序列。在此模型当中, 影响指每个实体 $c$ 在 $t$ 时刻的隐藏状态 $h_{t}^{(c)}$ 与所有实体在 $t-1$ 时刻的隐藏 状态 $h_{t-1}^{(1)}, \ldots, h_{t-1}^{(C)}$ 之间的状态依赖关系 ${ }^{[9]}$, 表示为:

$$
\left.\left.\operatorname{Prob}\left(h_{t}^{\left(c^{\prime}\right)}\right) h_{t-1}^{(1)}, \ldots, h_{t-1}^{c}\right)=\sum_{c \in\{1, \ldots, c)} R^{c^{\prime}, c} \times \operatorname{Prob}\left(h_{t}^{\left(c^{\prime}\right)}\right) h_{t-1}^{c}\right) \quad \text { (1) }
$$

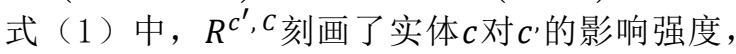
被称之为影响矩阵, 且它是一个 $\mathrm{C} \times \mathrm{C}$ 的行随机矩阵。 $\operatorname{Prob}\left(h_{t}^{\left(c^{(}\right)} \mid h_{t-1}^{C}\right)$ 刻画了不同实体隐藏状态之间的转 移概率。一般情况下, 对于系统中的每个实体 $c^{\prime}$, 存 在 $C$ 个不同的隐藏状态概率矩阵来刻画实体 $c^{\prime}$ 与 $c=$ $1, \cdots, C$ 之间的状态转移, 但可以将其替换为两个 矩阵 $E^{c}$ 和 $F^{c}$ 来加以简化 ${ }^{[10]}$ 。 


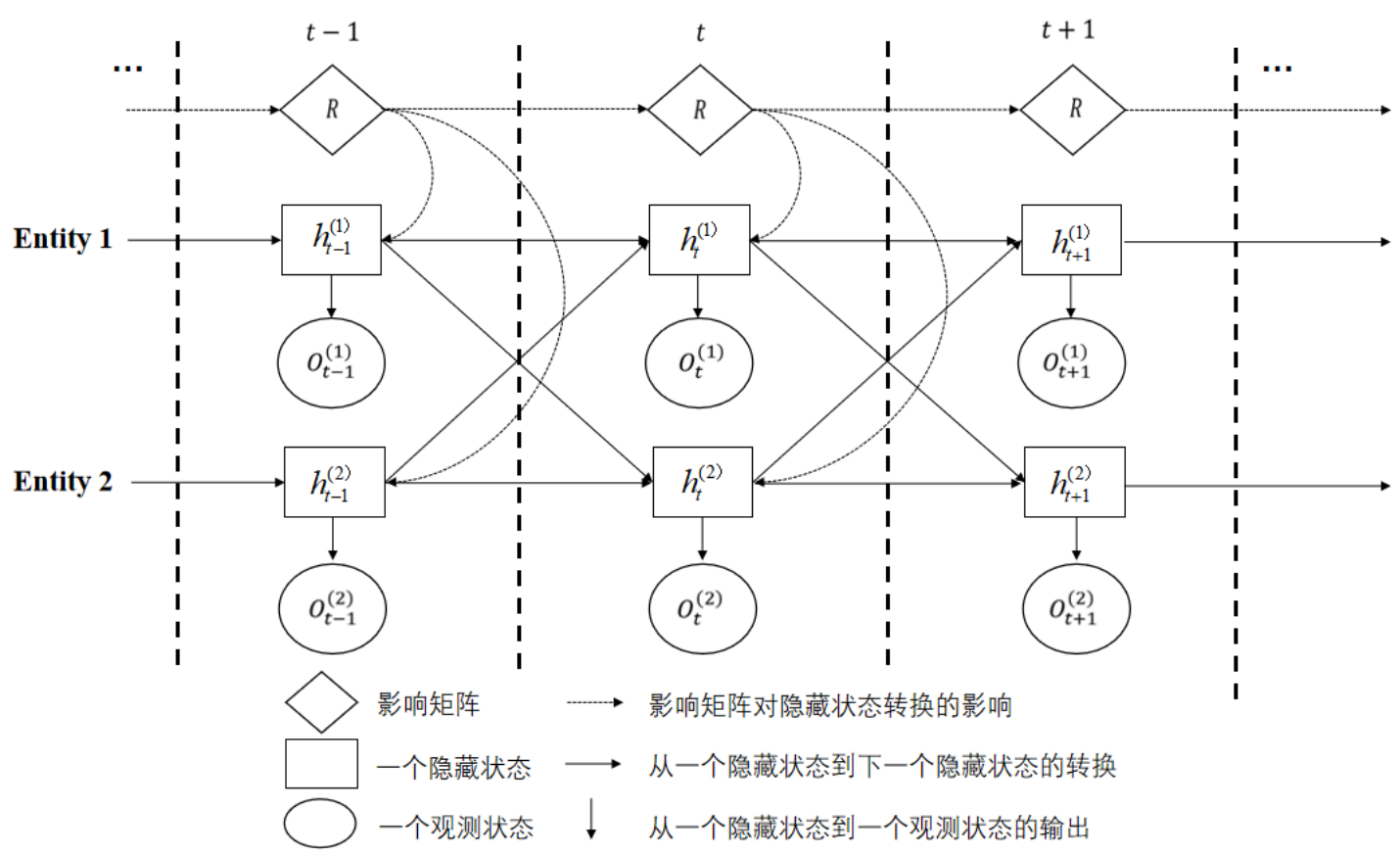

图 1 动态影响模型示意图

为了更好地对结果进行分析，描述实体之间的 交互影响关系，本文借鉴 Diebold 等的分析思路, 定义了以下评价指标对影响矩阵进行分析和评价 ${ }^{[11]}$ 。

1. 总影响指数: 衡量模型内所有市场在样本期 间交互影响强度的指标。计算公式如下:

$$
\text { Total }=\frac{\sum_{i, j=1}^{C} r_{i j}, i \neq j}{\sum_{i, j=1}^{C} r_{i j}}
$$

其中, $r_{i j}$ 是影响矩阵 $R$ 中处于第 $i$ 行第 $j$ 列的元素。

2. 定向接受指数：衡量系统内其他所有市场对 特定实体 $c$ 的影响强度的指标。计算公式如下：

$$
\text { Influ }_{\text {others } \rightarrow c}=\sum_{j=1}^{C}{ }_{j \neq i} r_{i j}, j \neq 1
$$

3. 定向给予指数: 衡量系统内特定实体 $c$ 对其他 所有市场的影响强度的指标。计算公式如下:

$$
\text { Influ } \text { I } \rightarrow \text { thers }=\sum_{i=1}^{C} r_{i \neq j} r_{i j}, i \neq 1
$$

4. 净影响指数: 定向给予指数与定向接受指数 之差。

$$
\text { Net }=\text { Infl } u_{c \rightarrow \text { others }}-\text { Influ } u_{\text {others } \rightarrow c}
$$

5. 成对影响指数: 衡量系统中两个特定实体之 间交互影响关系的指标。计算公式如下:

\section{3. 实证研究与结果分析}

\section{1. 样本数据的选择}

本文选取沪深 300 指数、中债 10 年期国债收益 率和沪深 300 期货指数作为中国股市、债市和期货 市场价格指数代表; 选取恒生指数、香港 10 期国债 收益率和恒生期货指数作为香港股市、债市和期货 市场价格指数代表; 选取标准普尔 500 指数、美国
10 年期国债收益率和标准普尔 500 期货指数作为美 国股市、债市和期货市场价格指数代表。样本期为 2011 年 3 月 1 日至 2021 年 2 月 28 日，删除由节日 差异造成的缺失值, 共计 2299 组数据, 所有数据来 源于 wind 数据库。各收益率采取的计算公式为:

$$
R_{i, t}=100 \times \ln \left(P_{i, t} / P_{i, t-1}\right)
$$

其中, $R_{i, t}(i=1,2, \ldots, 9)$ 表示金融市场 $i$ 在 $t$ 时刻 的收益率; $P_{i, t}$ 表示金融市场 $i$ 在 $t$ 时刻的收盘价。

\section{2. 运行环境的搭建及参数设定}

本文选用 Matlab 计算各个金融市场价格指数间 的影响矩阵，分析中国、香港和美国的股市、债市 和期货市场之间的交互影响关系。在本文中，用动 态影响模型对研究问题建模，实体就是各个金融市 场, 即参数 nAgents 设置为 9 ; 观测状态是各个金 融市场的收益率，由于是连续的时间序列，所以参 数 moutputs 设置为 1 。通过 BIC 准则确定出表示隐 藏状态个数的参数 nStates 为 3 , 即指数上涨、震 荡和下跌。在本模型当中，本文确定的最优迭代次 数为 125 。考虑到机器学习算法每次训练结果的随 机性, 本文将 20 次训练结果的均值作为最终结果。

\section{3. 实证分析}

将各个金融市场价格指数收益率时间序列数据 以. mat 的文件格式导入 Matlab 软件，可以得到如 图 2 所示的影响矩阵图, 根据影响矩阵分析评价指 标，可以得到如表 1 所示的九个金融市场价格指数 的交互影响表，表中未加粗的数据表示其所对应的 列所表示的市场对所对应的行所表示的市场的影响。 


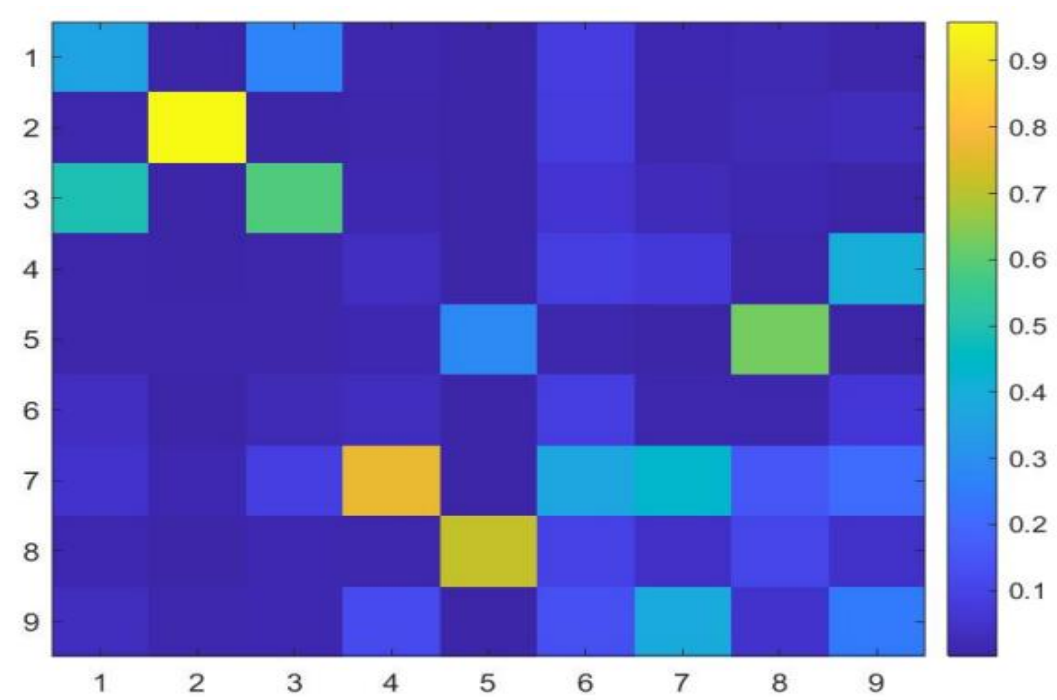

$$
\begin{aligned}
& \text { 1一中股 } \\
& \text { 2-中债 } \\
& \text { 3-中期 } \\
& \text { 4一港股 } \\
& \text { 5一港债 } \\
& \text { 6一港期 } \\
& \text { 7一美股 } \\
& \text { 8一美债 } \\
& \text { 9-美期 }
\end{aligned}
$$

图 2 影响矩阵

表 1 各个金融市场间的交互影响表（\%)

\begin{tabular}{|c|c|c|c|c|c|c|c|c|c|c|}
\hline & 中股 & 中债 & 中期 & 港股 & 港债 & 港期 & 美股 & 美债 & 美期 & $\begin{array}{c}\text { 定向接受指 } \\
\text { 数 }\end{array}$ \\
\hline 中股 & 29.68 & 4.05 & 40.27 & 2.64 & 1.66 & 1.92 & 9.94 & 3.00 & 6.83 & $\mathbf{7 0 . 3 2}$ \\
\hline 中债 & 0.60 & 94.91 & 0.33 & 0.67 & 0.39 & 0.77 & 0.74 & 0.40 & 1.18 & $\mathbf{5 . 0 9}$ \\
\hline 中期 & 32.99 & 2.07 & 46.55 & 1.46 & 1.21 & 2.39 & 7.18 & 2.00 & 4.14 & $\mathbf{5 3 . 4 5}$ \\
\hline 港股 & 2.07 & 3.98 & 2.26 & 1.94 & 1.41 & 2.10 & 39.95 & 5.13 & 41.16 & $\mathbf{9 8 . 0 6}$ \\
\hline 港债 & 0.03 & 0.17 & 0.09 & 0.21 & 10.92 & 0.16 & 0.40 & 87.58 & 0.43 & $\mathbf{8 9 . 0 8}$ \\
\hline 港期 & 3.46 & 4.17 & 2.44 & 2.78 & 2.46 & 3.11 & 43.07 & 5.36 & 33.15 & $\mathbf{9 6 . 8 9}$ \\
\hline 美股 & 1.58 & 1.67 & 1.49 & 10.06 & 0.67 & 12.40 & 25.78 & 3.20 & 43.16 & $\mathbf{7 4 . 2 2}$ \\
\hline 美债 & 0.69 & 0.87 & 0.67 & 0.73 & 29.68 & 1.10 & 4.22 & 59.64 & 2.40 & $\mathbf{4 0 . 3 6}$ \\
\hline 美期 & 1.77 & 2.23 & 1.12 & 16.66 & 0.64 & 13.08 & 22.53 & 2.56 & 39.41 & $\mathbf{6 0 . 5 9}$ \\
\hline 定向给予指 & $\mathbf{4 3 . 1 9}$ & $\mathbf{1 9 . 2 1}$ & $\mathbf{4 8 . 6 7}$ & $\mathbf{3 5 . 2 0}$ & $\mathbf{3 8 . 1 3}$ & $\mathbf{3 3 . 9 2}$ & $\mathbf{1 2 8 . 0 2}$ & $\mathbf{1 0 9 . 2 4}$ & $\mathbf{1 3 2 . 4 6}$ & \\
\hline 数 & & & & & & & & & & $\mathbf{9 6 3}$ \\
\hline 净影响指数 & $\mathbf{- 2 7 . 1 2}$ & $\mathbf{1 4 . 1 2}$ & $\mathbf{- 4 . 7 7}$ & $\mathbf{- 6 2 . 8 6}$ & $\mathbf{- 5 0 . 9 4}$ & $\mathbf{- 6 2 . 9 7}$ & $\mathbf{5 3 . 8 0}$ & $\mathbf{6 8 . 8 8}$ & $\mathbf{7 1 . 8 8}$ & \\
\hline 总影响指数 & $\mathbf{7 2 . 8 8}$ & $\mathbf{1 1 4 . 1 2}$ & $\mathbf{9 5 . 2 3}$ & $\mathbf{3 7 . 1 4}$ & $\mathbf{4 9 . 0 6}$ & $\mathbf{3 7 . 0 3}$ & $\mathbf{1 5 3 . 8 0}$ & $\mathbf{1 6 8 . 8 8}$ & $\mathbf{1 7 1 . 8 8}$ & $\mathbf{6 5 . 3 4}$ \\
\hline
\end{tabular}

对角线数据表示可由自身冲击解释的部分, 从 表 1 数据来看, 不同地区不同金融市场前期波动对 自身的影响不一致, 但所有市场对自身波动的影响 平均值也占到了 $34.66 \%$ 。就地区而言, 中国和美国 的金融市场都较易受到自身前期市场的影响, 而香 港的金融市场前期波动对自身的影响非常小，这与 各国的资本市场开放程度关系较为密切。就具体的 金融市场而言, 中国债券市场对自己的影响达到了 94. 91\%, 说明它最易受到自身前期变化的影响, 其 他市场的波动对其影响非常小, 从表 1 中的数据也 可以看出相对于股市和期货市场, 债券市场的前期 波动对自身的影响都较强, 这与债券市场的特点也 是相符的; 香港股票市场对自己的影响只有 $1.94 \%$, 说明它是最不易受到自身前期变化影响的市场。

从定向给予指数来看, 美国的三个金融市场对 其他金融市场的交互影响程度都较大, 均达到了 $105 \%$ 。这主要是由美国国际金融中心地位决定的, 美国国际金融中心地位使得大量经济金融信息在这 里交互传导, 从而使得美国金融市场对其他国家和 地区金融市场的影响程度较强, 除此之外, 美国资 本市场的完全开放也加强了这种强度。从定向接受
指数来看, 香港金融市场受其他两国金融市场的交 互影响程度较大, 均达到了 $85 \%$ 。这主要是由于香 港虽是国际金融中心，但其实体经济总量和资本市 场规模相较于中国和美国都较小，在国际金融环境 发生变化时较易被其他两国金融市场的波动所影响。

从净影响指数来看，中国股市、中国期货市场、 香港股市、香港债市和香港期货市场的净影响指数 为负, 说明它们是各个金融市场交互影响的被动接 受者; 中国债市、美国股市、美国债市和美国期货 市场的净影响指数为正, 说明它们是各个金融市场 交互影响的主动发出者。整体而言, 美国金融市场 均是各个金融市场价格指数交互影响的主动发出者, 这与前面分析得到的结论是一致的, 中国债市由于 受自身前期波动影响较强, 在整个资本市场中表现 出较为稳定的特点, 使得其他金融市场波动对其影 响非常小, 最终处于一个主动发出者的角色。

成对影响指数的计算结果如表 2 所示, 右上表 格表示首行各变量对首列各变量的影响程度, 成对 影响指数只是对于两个市场间的交互影响进行分析, 从表格中我们可以发现, 表格关于对角线互为相反 数, 即相对应的一组数据表示两者之间的影响指数。 
表 2 各个金融市场价格指数波动率成对影响指数表

\begin{tabular}{|c|c|c|c|c|c|c|c|c|c|}
\hline & 中股 & 中债 & 中期 & 港股 & 港债 & 港期 & 美股 & 美债 & 美期 \\
\hline 中股 & & 3.45 & 7.29 & 0.57 & 1.63 & -1.54 & 8.35 & 2.32 & 5.06 \\
\hline 中债 & -3.45 & & -1.74 & -3.32 & 0.22 & -3.40 & -0.92 & -0.47 & -1.04 \\
\hline 中期 & -7.29 & 1.74 & & -0.80 & 1.12 & -0.05 & 5.70 & 1.33 & 3.02 \\
\hline 港股 & -0.57 & 3.32 & 0.80 & & 1.20 & -0.68 & 29.89 & 4.40 & 24.50 \\
\hline 港债 & -1.63 & -0.22 & -1.12 & -1.20 & & -2.30 & -0.27 & 57.90 & -0.22 \\
\hline 港期 & 1.54 & 3.40 & 0.05 & 0.68 & 2.30 & & 30.66 & 4.26 & 20.07 \\
\hline 美股 & -8.35 & 0.92 & -5.70 & -29.89 & 0.27 & -30.66 & & -1.02 & 20.64 \\
\hline 美债 & -2.32 & 0.47 & -1.33 & -4.40 & -57.90 & -4.26 & 1.02 & & -0.16 \\
\hline 美期 & -5.06 & 1.04 & -3.02 & -24.50 & 0.22 & -20.07 & -20.64 & 0.16 & \\
\hline
\end{tabular}

为了更加直观地展现各个金融市场间的交互影 响关系, 本文用网络关系图表示 (如图 3 左), 其中 节点的大小表示影响强度, 节点的颜色表示对外影 响的意愿, 节点之间的连线表示该点与其他点的交 互状况, 两节点之间箭头的方向表示两市场之间的 影响交互方向, 即颜色深浅和粗细代表了与其他点 互动的意愿程度, 箭头越粗代表的金融市场的影响 强度越大。通过图形可看出, 美国股市、债市和期 货市场是交互影响的主要发出者, 香港股市、债市 和期货市场是主要接受者。美国债市和香港债市的 相互影响强度较大, 相较中国, 美国金融市场与香 港金融市场的相互影响强度要高。

65. 34\%, 即在导致这九个金融市场价格指数收益率 波动的因素中, 约有 $65.34 \%$ 的因素是由各个金融市 场价格指数的交互影响导致的, 即各个金融市场价 格指数之间的交互影响关系较为密切, 任何金融市 场价格指数存在波动都会对其他金融市场产生影响。 为了描述不同时点各个金融市场价格指数收益率之 间的交互影响关系, 本文采用滑动窗口思想, 将滑 动窗口宽度设置为 120 天, 步长设置为 10 天, 得到 了总影响指数在样本期间的时间序列, 如图 3 右。 从图中可以看出, 各个金融市场间的交互影响关系 具有时变性, 总影响指数在 $60 \%-70 \%$ 之间表面样本 期内各个市场之间的关系都比较密切且稳定。

从表 1 右下角的总影响指数来看, 其值为
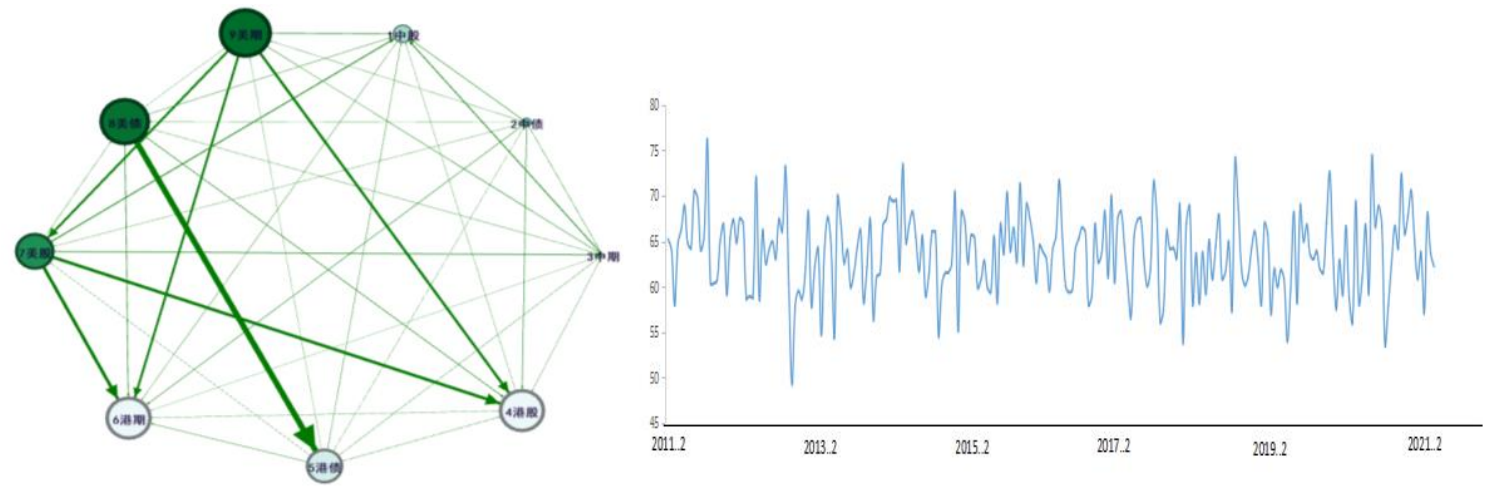

图 3 网络关系图(左)和 总影响指数时序图 (右)

\section{4. 不同大环境背景下对各个金融市场间交互 影响的分析}

2018 年是全球经济动荡的一年, 美国在特朗普 的领导之下, 向各国挑起贸易争端, 在 2018 年 3 月 23 日凌晨与中国的贸易冲突升级, 在同年 6 月份中 美贸易战正式爆发。中美贸易战爆发之后, 全球市 场对此产生了剧烈的反应, 接下来本文将以 2018 年 6 月 1 日为界比较分析中美贸易战前后一年中国、 香港和美国的股市、债市和期货市场的网络关系图 的具体变化。通过图 5 中的对比, 在其他设置条件 相同的前提之下, 右图的箭头颜色比左图的明显更 粗, 这说明在中美贸易战爆发之后, 各个金融市场 价格指数收益率之间的交互影响关系变得更加密切, 这与中美贸易战对市场产生影响的现实状况一致,
具体来看, 美国股票市场在整个网络关系中的角色 显得十分主动, 结合实际情况来看, 这是由于特朗 普的贸易保护主义措施加剧了投资者的恐惧, 从而 导致股市动荡进而对其他市场产生影响, 而对于中 国而言, 在中美贸易战中, 中国本就属于应战者, 并非战争的挑起者, 这也使得中国市场在整个网络 关系图中处于影响接受者的角色, 所以无论是之前 还是之后, 中国金融市场在与美国金融市场的交互 关系当中, 都是影响的接受者, 而香港市场在中美 贸易战之后股票市场也受到了其他金融市场严重的 影响。从图 5 中中国和香港金融市场的节点对比可 以明显地发现, 中美贸易战之后中国成为了主要的 影响接受者, 这主要是由于中国和美国是中美贸易 战债市和期货市场之间的交互影响程度都大大增强, 香港与中国的交互影响关系也明显增强。 

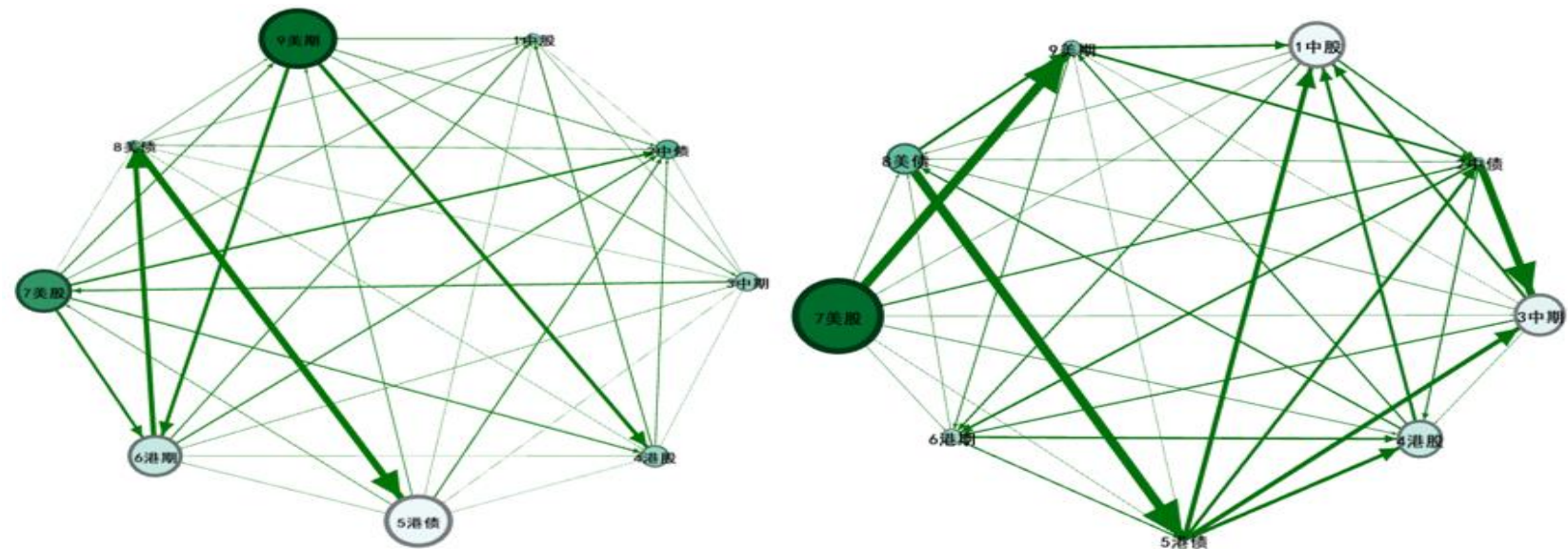

图 5 中美贸易战前后（左为前，右为后）网络关系图

\section{5. 结论}

本文运用动态影响模型和五种影响矩阵评价指 标对中国、香港和美国的股市、债市和期货市场指 数的交互影响进行研究, 得到如下研究结论：（1） 各个金融市场价格指数之间的交互影响关系比较密 切, 且具有时变性。(2) 中国债券市场是最容易受 自身前期变化影响的金融市场, 香港股票市场是最 不容易受自身前期变化影响的金融市场。（3）美国 股市、美国债市和美国期货市场对其他金融市场的 交互影响程度较大, 是交互影响的主要的发出者, 香港股市、香港债市和香港期货市场受到其他金融 市场的影响较大, 是交互影响主要的接受者。(4) 无论是在中美贸易战前还是后, 美国都是三者当中 交互影响的最主要的主动发出者, 且对香港的影响 程度强于中国, 而在中国和香港这对关系中, 中国 处于主动发出者的角色。（5）在中美贸易战之后, 中国和香港的金融市场互动关系增强。

\section{REFERENCES}

[1] Cui J.X,Zou H.W. (1994) Research on the Spillover Effect of High-Order Moments Risk in China's Stock Market .J. Systems Science and Mathematical Sciences,40(07):1178-1204.

[2] Wang L.F. (2021) Analysis on the Fluctuation Effect among China's Financial Markets.J. Modern Business Trade

[3] Wang L,Li B.X,Ma X.T ,Zheng Y.(2021) The Spillover Effect and Persistence of Price Fluctuation between China's Crude Oil Futures and International Crude Oil Futures: A Study Based on the BEKK-MGARCH Model.J/OL. Systems Engineering:1-22. http://kns.cnki.net/kcms/detail.

[4] Yuan W,Wang P.H.(2020) An Empirical Study on Information Spillover Effect of Sino-US Financial Markets.J. Finance Forum, 25(07):43-52.

[5] Ni Z.X,Wang F.Y.(2014) A Study on the Spillover
Effect of US Monetary Policy on BRICS Countries - A Comparative Analysis Based on VAR Model.J. South Finance (04):20-25.

[6] Wang S,Zhao C.C.(2020) The Transmission Mechanism between RMB Exchange Rate and Stock Price: An Empirical Test Based on DCCGARCH Model.J. Industrial Technology Economy, 39(04):54-62.

[7] Reng Y.H,Zhao W.R,Luo L.Q.(2020) Research on the Characteristics of Risk Spillover Network in Stock Market Based on Copula Function.J.Forum on Statistics and Information,35(08):53-63.

[8] Wei X,Ning X.P,Li F.Y.(2020) An Empirical Test on the Relationship between non-deliverable forward Exchange Rate and RMB Exchange Rate in Mainland China .J. Management Review,32(11):81-91.

[9] Pentland,A,Wang X.F,Wang R.(2015) Smart Society: Big Data and Social Physics. Zhejiang people's press,Hangzhou.

[10] Pan W, Dong W, Cebrian M , Kim T,Fowler J.H,Pentland A. (2012)Modeling Dynamical Influence in Human Interaction: Using data to make better inferences about influence within social systems.J. IEEE Signal Processing Magazine, 29(2):77-86.

[11] Francis X Diebold, Kamil Yilmaz. (2012) Better to Give than to Receive: Predictive Measurement of Volatility Spillovers.J. International Journal of Forecasting, (28): 57-66. 